An ef $\mathrm{fi}$ ci ent 1,3 di pol ar cycl oaddi t $\mathrm{i}$ on bet ween ar omat i c sel enoal dehydes and nitrile oxi des or nitrile i mines: an easy access to sel eni um cont ai ni ng fi ve- member ed het er ocycl i c ring system

\begin{tabular}{|l|l|}
\hline 著者 & $\begin{array}{l}\text { Segi Nasahi to, Tanno Kat suhi ko, Koj i ma Nasum, } \\
\text { Honda M t sunor i , Nakaj i ma Tadashi }\end{array}$ \\
\hline $\begin{array}{l}\text { j our nal or } \\
\text { publ i cat i on t i t l e }\end{array}$ & Tet r ahedr on Let ter s \\
\hline vol une & 48 \\
\hline number & 13 \\
\hline page range & $2303-2306$ \\
\hline year & $2007-03-01$ \\
\hline URL & ht t p: //hdl . handl e. net /2297/3783 \\
\hline
\end{tabular}




\section{Graphical Abstract}

To create your abstract, type over the instructions in the template box below.

Fonts or abstract dimensions should not be changed or altered.

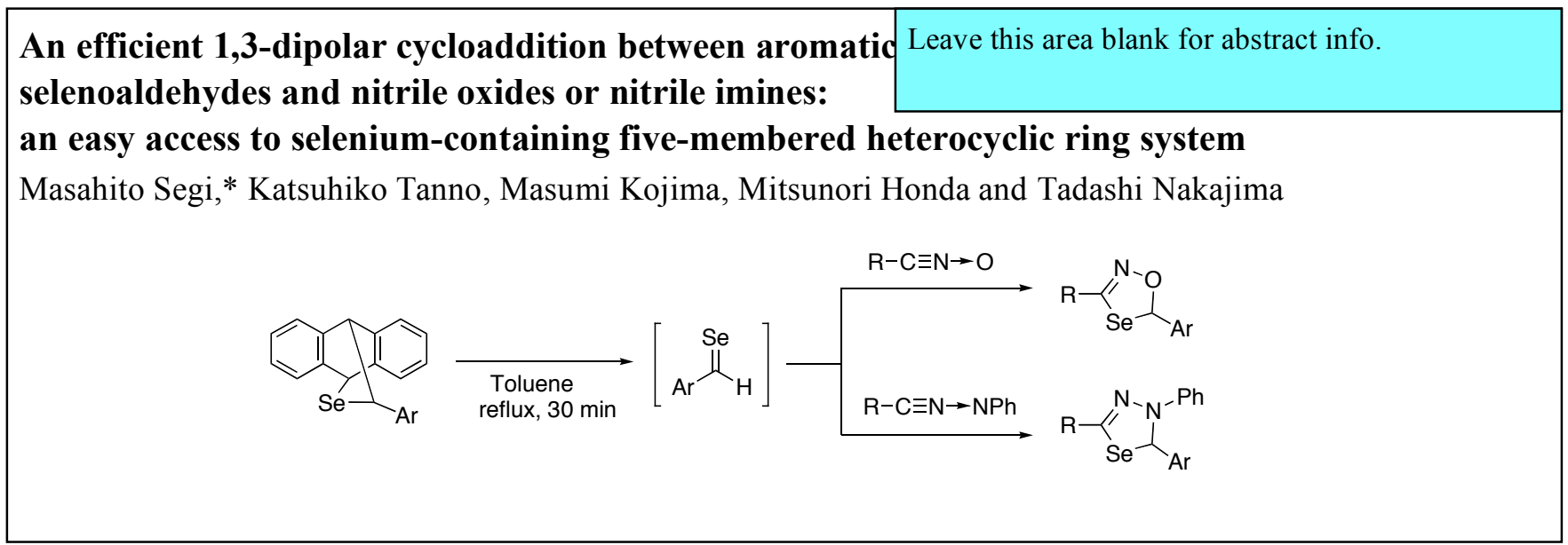




\title{
An efficient 1,3-dipolar cycloaddition between aromatic selenoaldehydes and nitrile oxides or nitrile imines: an easy access to selenium-containing five-membered heterocyclic ring system
}

\author{
Masahito Segi, * Katsuhiko Tanno, Masumi Kojima, Mitsunori Honda \\ and Tadashi Nakajima \\ Division of Material Sciences, Graduate School of Natural Science and Technology, Kanazawa University, \\ Kakuma-machi, Kanazawa 920-1192, Japan
}

Received xx January 2007

\begin{abstract}
Dipolar cycloaddition between aromatic selenoaldehydes, generated by thermal retro Diels-Alder reaction of anthracene cycloadducts, and nitrile oxides or nitrile imines proceeded efficiently to give the corresponding [3+2] cycloadducts as a single isomer in good yields, being 1,4,2-oxaselenazoles or 1,3,4-selenadiazoles, respectively.
\end{abstract}

(C) 2007 Elsevier Ltd. All rights reserved.

Selenocarbonyl compounds are very reactive selenium analogues of carbonyl compounds and play an increasingly important role in organic synthesis. ${ }^{1}$ Among them selenoaldehydes and selenoketones have been well recognized to serve as significant $2 \pi$ dienophiles in cycloaddition reactions. ${ }^{2}$ In this context, cycloaddition of the reactive selenoaldehydes is an important approach to the preparations of selenium-containing heterocycles which have been recently paid much attention because of their interesting reactivities ${ }^{3}$ and their potential biological applications. ${ }^{4}$ In the course of our studies for generation and reaction of reactive selenoaldehydes, ${ }^{5}$ we have developed an efficient method for the generation of selenoaldehydes under neutral conditions via thermal retro Diels-Alder reaction of selenoaldehyde-anthracene cycloadducts 1 that were easily synthesized in good yields from the reaction of the corresponding aldehydes with $\left(\mathrm{Me}_{2} \mathrm{Al}\right)_{2} \mathrm{Se}$ in the presence of anthracene (Scheme 1). ${ }^{6} \mathrm{We}$ have already reported the reactions of selenoaldehydes with oxygen-functionalized conjugated dienes, ${ }^{7}$ 2methoxyfuran, ${ }^{8}$ and 5 -ethoxyoxazoles ${ }^{9}$ using the above retro Diels-Alder protocol.

Keywords: Selenoaldehyde; 1,3-Dipolar cycloaddition; Nitrile oxide; Nitrile imine; Selenium-containing heterocycle.

* Corresponding author. Tel.: +81 76234 4787; fax: +81 76234 4800; email: segi@t.kanazawa-u.ac.jp

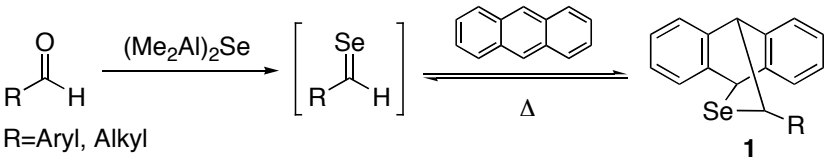

Scheme 1. Generation of selenoaldehydes.

On the other hand, 1,3-dipolar cycloaddition has been considered to be the most useful method for the construction of five-membered heterocyclic ring systems containing one or more heteroatoms. ${ }^{10}$ Selenoaldehydes serve as an excellent dienophile as mentioned above and would also have a high potential as a good dipolarophile. Indeed 1,3-dipolar cycloaddition between selenoaldehydes and a stable nitrile oxide (2,4,6-trimethylbenzonitrile $\mathrm{N}$ oxide) has been already reported. ${ }^{11}$ However, to our knowledge, up to date there are no reports regarding 1,3dipolar cycloaddition of other 1,3-dipoles with selenoaldehydes. We have carried out the reaction of selenobenzaldehyde, generated by our thermal retro DielsAlder reaction of $1(\mathrm{R}=\mathrm{Ph})$, with some popular 1,3-dipoles such as nitrones, nitrile oxides, azomethine ylides, and nitronates, but only reaction with nitrile oxides gave the corresponding $[3+2]$ cycloadducts of the selenoaldehyde in good yields as isolable compounds. This result may indicate that efficient $[3+2]$ cycloaddition reaction of selenoaldehydes can be achieved by using a linear-type 1,3dipole such as nitrile oxide. In this paper we describe an efficient 1,3-dipolar cycloaddition between aromatic selenoaldehydes and nitrile oxides or nitrile imines as a 
linear-type 1,3-dipole to afford selenium-containing fivemembered ring products, 1,4,2-oxaselenazoles or $1,3,4-$ selenadiazoles, respectively.

Most nitrile oxides are short-lived and reactive species, and easily dimerize, rearrange, or polymerize. ${ }^{12}$ At first, we used a stable aromatic nitrile oxide, 2,4,6trimethylbenzonitrile $\mathrm{N}$-oxide, ${ }^{13}$ easily prepared through two steps from 2,4,6-trimethylbenzaldehyde. The toluene solution of $1(\mathrm{R}=\mathrm{Ph})$ and the stable nitrile oxide was heated at reflux for $30 \mathrm{~min}$ to give the desired [3+2] cycloadduct (2a) as a single isomer in $85 \%$ yield (Eq. (1)). The structure of this adduct was supported by the analysis of the NMR and mass spectra. ${ }^{14}$ In particular, regiochemistry of the cycloadduct was confirmed by the mass spectrum which showed a fragment peak at $\mathrm{m} / \mathrm{z} 225$ with an isotope pattern involving one selenium atom, corresponding to [M$\mathrm{PhCHO}^{+}$as shown in Scheme 2. This indicates that a carbon-oxygen bond exists in the obtained cycloadduct, being 1,4,2-oxaselenazole.

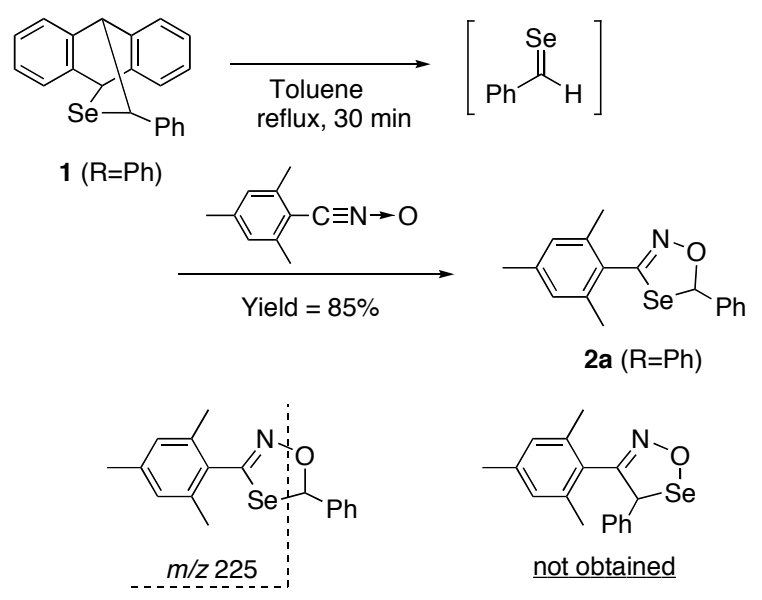

Scheme 2.

Several anthracene cycloadducts with an aromatic substituent were similarly treated with 2,4,6trimethylbenzonitrile $N$-oxide in toluene at reflux for 30 min to give the corresponding [3+2] cycloadducts $(2)^{14}$ as a single isomer in good yields. The results are shown in Table 1. Mass spectra of all cycloadducts showed a same fragment peak pattern at $\mathrm{m} / \mathrm{z} 225$ that is corresponding to $[\mathrm{M}-\mathrm{ArCHO}]^{+}$, which indicates all obtained cycloadducts have 1,4,2-oxaselenazole skeleton. Anthracene cycloadducts having an aliphatic group did not undergo retro Diels-Alder reaction at toluene reflux temperature, but at over $150^{\circ} \mathrm{C}$, the generation of aliphatic selenoaldehydes was efficient. Thus, we examined the reaction of $1(\mathrm{R}=n-\mathrm{Pr}$ and $\mathrm{CH}_{2} \mathrm{CH}_{2} \mathrm{Ph}$ ) with the nitrile oxide at $160^{\circ} \mathrm{C}$ in toluene, but no isolable cycloadducts could be obtained from the reaction mixture.

Next we planned the reaction of selenoaldehydes with unstable nitrile oxides which are in situ formed via dehydrochlorination of hydroximoyl chlorides with triethylamine as a base. $^{12}$ Generally, this
Table 1. 1,3-Dipolar cycloaddition between aromatic selenoaldehydes and 2,4,6-trimethylbenzonitrile $N$-oxide

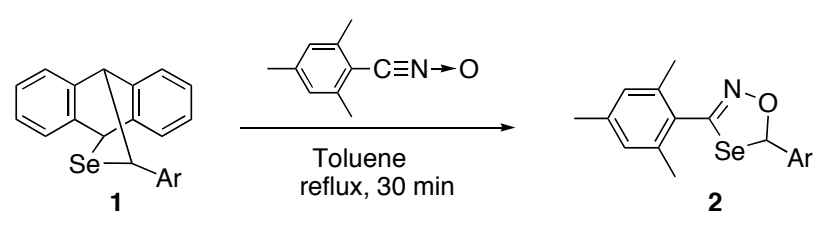

\begin{tabular}{clcc}
\hline Entry & Ar & Cycloadduct & ${\text { Yield }(\%)^{\mathrm{a}}}^{\mathrm{a}}$ \\
\hline 1 & $\mathrm{Ph}$ & $\mathbf{2 a}$ & 85 \\
2 & $p-\mathrm{ClC}_{6} \mathrm{H}_{4}$ & $\mathbf{2 b}$ & 74 \\
3 & 1-naphthyl & $\mathbf{2 c}$ & 96 \\
4 & $p-\mathrm{CF}_{3} \mathrm{C}_{6} \mathrm{H}_{4}$ & $\mathbf{2 d}$ & 85 \\
5 & $p-\mathrm{CH}_{3} \mathrm{C}_{6} \mathrm{H}_{4}$ & $\mathbf{2 e}$ & 65 \\
\hline
\end{tabular}

a Isolated yield.

dehydrochlorination has to be carried out in the presence of the dipolarophile, since the lifetime of the reactive nitrile oxide is too short for isolation. However selenoaldehydes, being a dipolarophile in this research, are also much more reactive and very unstable. Accordingly, both selenoaldehyde and nitrile oxide must be efficiently in situ generated in the reaction mixture simultaneously. After several trials under some different conditions, we found a following reaction procedure for an efficient 1,3-dipolar cycloaddition. After 6 equiv of triethylamine was added to a toluene solution involving $1(\mathrm{R}=\mathrm{Ar})$ and 3 equiv of aromatic hydroximoyl chloride at room temperature, the reaction vessel was immediately placed for $30 \mathrm{~min}$ into oil bath preheated at $110^{\circ} \mathrm{C}$. The results are summarized in Table 2. In all cases, an efficient 1,3-dipolar cycloaddition proceeded to give the desired [3+2] cycloadducts $(3), 1,4,2-$ oxaselenazoles, as a single regioisomer in good yields. ${ }^{15}$ They were very stable at room temperature in the atmosphere. The regiochemistry of the obtained [3+2] cycloadducts was the same as the case using 2,4,6trimethylbenzonitrile $N$-oxide, that is, the mass spectra of

Table 2. 1,3-Dipolar cycloaddition between aromatic selenoaldehydes and unstable nitrile oxides

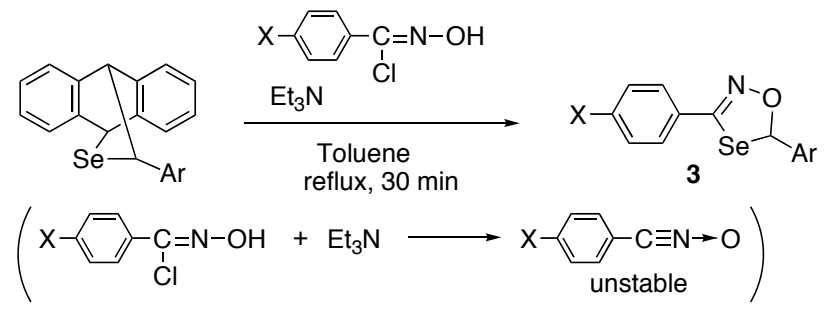

\begin{tabular}{cllcl}
\hline Entry & $\mathrm{Ar}$ & $\mathrm{X}$ & Cycloadduct & Yield (\%) $^{\mathrm{a}}$ \\
\hline 1 & $\mathrm{Ph}$ & $\mathrm{MeO}$ & $\mathbf{3 a}$ & 67 \\
2 & 1-naphthyl & $\mathrm{MeO}$ & $\mathbf{3 b}$ & 67 \\
3 & $p-\mathrm{CF}_{3} \mathrm{C}_{6} \mathrm{H}_{4}$ & $\mathrm{MeO}$ & $\mathbf{3 c}$ & 94 \\
4 & $p-\mathrm{CF}_{3} \mathrm{C}_{6} \mathrm{H}_{4}$ & $\mathrm{H}$ & $\mathbf{3 d}$ & 65 \\
5 & $\mathrm{Ph}$ & $\mathrm{Cl}$ & $\mathbf{3 e}$ & 76 \\
6 & $p-\mathrm{CF}_{3} \mathrm{C}_{6} \mathrm{H}_{4}$ & $\mathrm{Cl}$ & $\mathbf{3 f}$ & 58 \\
\hline
\end{tabular}

a Isolated yield. 
the cycloadducts showed a fragment peak corresponding to $[\mathrm{M}-\mathrm{ArCHO}]^{+}$.

The reaction of selenoaldehydes with unstable aliphatic nitrile oxides did not give a satisfactory result, but the use of a stable aliphatic nitrile oxide, 2,2,3triphenylpropanenitrile $\mathrm{N}$-oxide, ${ }^{16}$ resulted in the excellent result as shown in Eq. 2. In this case, the [3+2] cycloadduct was obtained in excellent yield as a single isomer with the same regiochemical result as mentioned above.

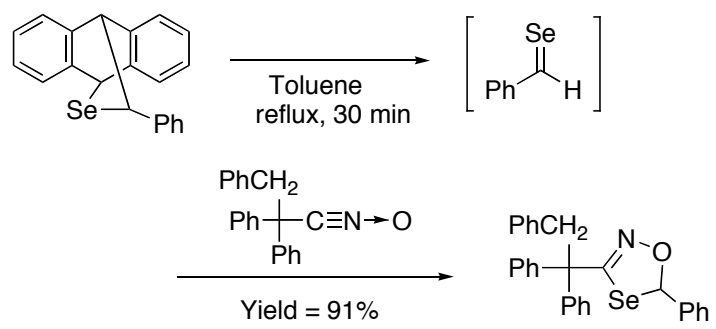

Nitrile imines are also recognized as a linear-type 1,3dipole and in situ generated via dehydrochlorination of hydrazonoyl chlorides with triethylamine. ${ }^{12 \mathrm{a}} \mathrm{We}$ have examined 1,3-dipolar cycloaddition between aromatic selenoaldehydes and aromatic $N$-phenyl nitrile imines, using a similar reaction procedure as the aforementioned 1,3-dipolar cycloaddition of nitrile oxides. The expected [3+2] cycloadducts (4), 1,3,4-selenadiazoles, were obtained as a single regioisomer in good yields and identified by the analysis of the NMR and mass spectra. ${ }^{17}$ The results are shown in Table 3. No significant fragment peak corresponding to [M-ArCHNPh $]^{+}$was observed in the mass spectra, but the signal corresponding to the eliminated fragment ion, [ArCHNPh $]^{+}$, appeared clearly with over $50 \%$ relative intensity in all cases. These seleniumcontaining five-membered heterocycles were also stable at room temperature in the atmosphere.

Table 3. 1,3-Dipolar cycloaddition between aromatic selenoaldehydes and $N$-phenyl nitrile imines

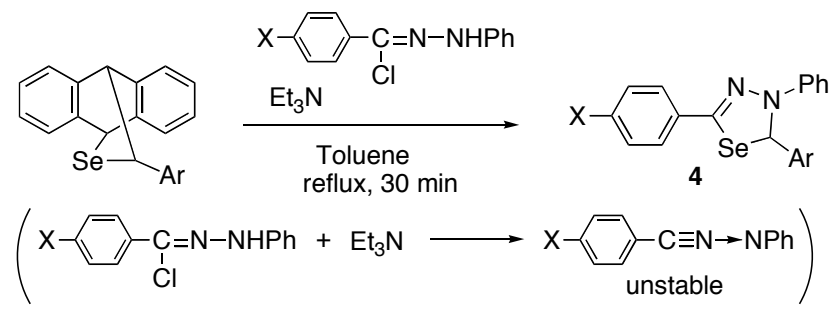

\begin{tabular}{cllcc}
\hline Entry & $\mathrm{Ar}$ & $\mathrm{X}$ & Cycloadduct & Yield (\%) $^{\mathrm{a}}$ \\
\hline 1 & $\mathrm{Ph}$ & $\mathrm{H}$ & $\mathbf{4 a}$ & 81 \\
2 & $p-\mathrm{MeOC}_{6} \mathrm{H}_{4}$ & $\mathrm{H}$ & $\mathbf{4 b}$ & 67 \\
3 & $p-\mathrm{CF}_{3} \mathrm{C}_{6} \mathrm{H}_{4}$ & $\mathrm{H}$ & $\mathbf{4 c}$ & 85 \\
4 & $\mathrm{Ph}$ & $\mathrm{NO}_{2}$ & $\mathbf{4 d}$ & 68 \\
5 & $p-\mathrm{CF}_{3} \mathrm{C}_{6} \mathrm{H}_{4}$ & $\mathrm{NO}_{2}$ & $\mathbf{4 e}$ & 87 \\
\hline
\end{tabular}

a Isolated yield.
In conclusion, we have demonstrated that regioselective 1,3-dipolar cycloaddition between aromatic selenoaldehydes, generated from 1 via thermal retro DielsAlder reaction, and nitrile oxides or nitrile imines proceeded efficiently to afford the [3+2] cycloadducts, 1,4,2-oxaselenazoles or 1,3,4-selenadiazoles, respectively, in good yields. This method offered a promising prospect for building up selenium-containing five-membered heterocyclic ring system. We are currently extending the scope of 1,3-dipolar cycloaddition between selenoaldehydes and other 1,3-dipoles. The results of our findings will be reported in due course.

\section{Acknowledgments}

This work was partially supported by a Grant-in-Aid for Scientific Research from the Ministry of Education, Science, Sports and Culture, Japan.

\section{References}

1. For reviews, see (a) Paulmier, C. In Selenium Reagents and Intermediates in Organic Synthesis; Baldwin, J. E., Ed.; Pergamon Press: Oxford, UK, 1986; pp. 58-83; (b) Guziec, F. S., Jr. In Organoselenium Chemistry; Liotta, D., Ed.; John Wiley \& Sons: New York, 1987; pp. 277-324; (c) Guziec, F. S., Jr. In The Chemistry of Organic Selenium and Tellurium Compounds; Patai, S., Ed.; John Wiley \& Sons: New York, 1987; Vol. 2, pp. 215-273; (d) Segi, M.; Nakajima, T. Yuki Gosei Kagaku Kyokaishi 1995, 53, 678-686; (e) Murai, T.; Kato, S. In Topics in Current Chemistry (Organoselenium Chemistry); Wirth, T., Ed.; Springer: Berlin, 2000; Vol. 208, pp. 177-199.

2. (a) Okazaki, R. Yuki Gosei Kagaku Kyokaishi 1988, 46, 11491163; (b) Tokitoh, N.; Okazaki, R. Pol. J. Chem. 1998, 72, 971-1000; (c) Guziec, L. J.; Guziec, F. S. Jr. In Comprehensive Organic Functional Group Transformations 2; Katritzky, A. R.; Taylor, R. J. K., Eds.; Elsevier: Amsterdam, 2005; pp. 397-417; For theoretical studies, see (d) Rohr, U.; Schatz, J.; Sauer, J. Eur. J. Org. Chem. 1998, 2875-2883; (e) Bachrach, S. M.; Jiang, S. J. Org. Chem. 1999, 64, 8248-8355; (f) Liao, H-Y.; Su, M-D.; Chu, S-Y. Chem. Phys. 2000, 261, 275-287; (g) Orlova, G.; Goddard, J. D. J. Org. Chem. 2001, 66, 4026-4035.

3. (a) Renson, M. In The Chemistry of Organic Selenium and Tellurium Compounds; Patai, S.; Rappoport, Z., Eds.; John Wiley \& Sons: New York, 1986; Vol. 1, pp. 399-516; (b) Litvinov, V. P.; Dyachenko, V. D. Russ. Chem. Rev. 1997, 66, 923-951.

4. (a) Wendel, A. Selenium in Biology and Medicine; SpringerVerlag: Berlin, 1989; (b) Burk, R. F. Selenium in Biology and Human Health; Springer-Verlag: New York, 1994.

5. (a) Segi, M.; Nakajima, T.; Suga, S.; Murai, S.; Ryu, I.; Ogawa, A.; Sonoda, N. J. Am. Chem. Soc. 1988, 110, 19761978; (b) Segi, M.; Takahashi, M.; Nakajima, T.; Suga, S.; Murai, S.; Sonoda, N. Tetrahedron Lett. 1988, 29, 6965-6968; (c) Segi, M.; Koyama, T.; Nakajima, T.; Suga, S.; Murai, S.; Sonoda, N. Tetrahedron Lett. 1989, 30, 2095-2098; (d) Segi, M.; Kato, M.; Nakajima, T. Tetrahedron Lett. 1991, 32, 74277430; (e) Li, G. M.; Segi, M.; Nakajima, T. Tetrahedron Lett. 1992, 33, 3515-3518; (f) Segi, M.; Takahashi, T.; Ichinose, H.; Li, G. M.; Nakajima, T. Tetrahedron Lett. 1992, 33, 7865- 
7868; (g) Li, G. M.; Niu, S.; Segi, M.; Zingaro, R. A.; Yamamoto, H.; Watanabe, K.; Nakajima, T.; Hall, M. B. J. Org. Chem. 1999, 64, 1565-1575.

6. Segi, M. Yuki Gosei Kagaku Kyokaishi 2003, 61, 661-669.

7. (a) Segi, M.; Yamamoto, H.; Hori, T.; Nakajima, T. Phosphorus, Sulfur Silicon Relat. Elem. 1998, 136, 137 \&138, 599-602; (b) Tomoda, S.; Zhang, J.; Kaneno, D.; Segi, M.; Zhou, A. Tetrahedron Lett. 2000, 41, 4597-4601.

8. Zhou, A.; Segi, M.; Nakajima, T. Tetrahedron Lett. 2003, 44, 1179-1182.

9. Segi, M.; Zhou, A.; Honda, M. Phosphorus, Sulfur, and Silicon 2005, 180, 1045-1049.

10. Padwa, A. 1,3-Dipolar Cycloaddition Chemistry; John Wiley \& Sons: New York, 1984; Vols. 1 and 2.

11. (a) Meinke, P. T.; Krafft, G. A. Tetrahedron Lett. 1987, 28, 5121-5124; (b) Meinke, P. T.; Krafft, G. A. J. Am. Chem. Soc. 1988, 110, 8671-8679; (c) Takeda, N.; Tokitoh, N.; Okazaki, R. Angew. Chem. Int. Ed. Engl. 1996, 35, 660-662; (d) Takeda, N.; Tokitoh, N.; Okazaki, R. Tetrahedron 1997, 53, 12167-12182.

12. (a) Caramella, P.; Grunanger, P. In 1,3-Dipolar Cycloaddition Chemistry; Padwa, A., Ed; John Wiley \& Sons: New York, 1984; Vol 1, pp. 291-392; (b) Torssell, K. B. G. Nitrile Oxides, Nitrones, and Nitronates in Organic Synthesis; VCH Publishers: New York, 1988; Chapter 2, pp. 55-74.

13. Grundmann, C.; Richter, R. J. Org. Chem. 1968, 33, 476-478.

14. Selected spectral data of $[3+2]$ Cycloadducts 2. Compound 2a; ${ }^{1} \mathrm{H}$ NMR $\left(400 \mathrm{MHz}, \mathrm{CDCl}_{3}\right): \delta 2.27(\mathrm{~s}, 3 \mathrm{H}), 2.29$ (s, 6H), $6.87(\mathrm{~s}, 2 \mathrm{H}), 7.30-7.57(\mathrm{~m}, 5 \mathrm{H}), 7.69(\mathrm{~s}, 1 \mathrm{H}) ;{ }^{13} \mathrm{C}$ NMR $(100$ $\left.\mathrm{MHz}, \mathrm{CDCl}_{3}\right): \delta 19.68,21.10,93.77,125.17,125.89,128.53$, $128.61,128.95,137.06,139.33,139.61,153.99 ;{ }^{77}$ Se NMR (76.2 MHz, $\mathrm{CDCl}_{3}$ ): $\delta 617.72$; Mass (EI mode): $\mathrm{m} / z$ (relative intensity) 51 (11.9), 77 (37.9), 105 (36.5), 130 (89.3), 145 (100), 225 (51.6), $331\left(\mathrm{M}^{+}, 0.7\right)$; HRMS calcd for $\left[\mathrm{C}_{10} \mathrm{H}_{11} \mathrm{NOSe}\left(\mathrm{M}^{+}-\mathrm{PhCHO}\right)\right] 225.0057$, found 225.0058 . Compound 2c; ${ }^{1} \mathrm{H}$ NMR (400 MHz, $\mathrm{CDCl}_{3}$ ): $\delta 2.19$ (s, 3H), $2.20(\mathrm{~s}, 6 \mathrm{H}), 6.79(\mathrm{~s}, 2 \mathrm{H}), 7.41-7.84(\mathrm{~m}, 7 \mathrm{H}), 8.22(\mathrm{~s}, 1 \mathrm{H})$; ${ }^{13} \mathrm{C}$ NMR $\left(100 \mathrm{MHz}, \mathrm{CDCl}_{3}\right): \delta 19.73,21.11,91.24,122.40$, 123.33 , $125.09,125.40,125.99,126.57,128.55,128.72$, $129.20,129.35,133.78,134.73,137.12,139.64,154.86 ;{ }^{77} \mathrm{Se}$ NMR (76.2 MHz, $\mathrm{CDCl}_{3}$ ): $\delta$ 615.61; Mass (EI mode): $\mathrm{m} / \mathrm{z}$ (relative intensity) 51 (14.3), 77 (28.1), 89 (27.5), 136 (81.3), 154 (100), 225 (27.7), 307 (16.1), $382\left(\mathrm{M}^{+}, 25.4\right)$; HRMS calcd for $\mathrm{C}_{21} \mathrm{H}_{20} \mathrm{NOSe}\left(\mathrm{M}^{+}\right)$382.0710, found 382.0712 . Compound 2d; ${ }^{1} \mathrm{H}$ NMR (400 MHz, $\left.\mathrm{CDCl}_{3}\right): \delta 2.25(\mathrm{~s}, 3 \mathrm{H})$, $2.27(\mathrm{~s}, 6 \mathrm{H}), 6.87(\mathrm{~s}, 2 \mathrm{H}), 765$ (br s, 4H), $7.72(\mathrm{~s}, 1 \mathrm{H}) ;{ }^{13} \mathrm{C}$ NMR $\left(100 \mathrm{MHz}, \mathrm{CDCl}_{3}\right): \delta 19.70,21.13,92.25,124.62$, $125.65,125.70,125.92,128.66,137.11,139.92,144.08$, $153.69 ;{ }^{77} \mathrm{Se} \mathrm{NMR}\left(76.2 \mathrm{MHz}, \mathrm{CDCl}_{3}\right): \delta 622.33$; Mass (EI mode): $\mathrm{m} / \mathrm{z}$ (relative intensity) 63 (18.7), 77 (37.9), 136 (84.8), 146 (35.4), 154 (100), 173 (27.1), 225 (50.3), $400\left(\mathrm{M}^{+}, 67.3\right)$; HRMS calcd for $\mathrm{C}_{18} \mathrm{H}_{17} \mathrm{~F}_{3} \mathrm{NOSe}\left(\mathrm{M}^{+}\right)$400.0427, found 400.0424 .
15. Typical experimental procedure: To an oven dried $30 \mathrm{~mL}$ round bottomed flask fitted with a reflux condenser were added an anthracene cycloadduct (1, $0.5 \mathrm{mmol})$, hydroximoyl chloride $(1.5 \mathrm{mmol})$, and toluene $(15 \mathrm{~mL})$ under an argon atmosphere. This mixture was then stirred until all of the solids dissolved at room temperature. Once a clear solution was obtained, triethylamine $(3.0 \mathrm{mmol})$ was added to the reaction mixture, and the reaction vessel was immediately placed into an oil bath preheated at $110^{\circ} \mathrm{C}$. After the heating at $110^{\circ} \mathrm{C}$ for $30 \mathrm{~min}$, the mixture was allowed to cool to room temperature and evaporated in vacuo. The resulting residue was purified by column chromatography on silica gel with 40:1 hexane / ethyl acetate to give the desired cycloadduct 3 as a single isomer. Selected spectral data of $[3+2]$ Cycloadducts 3. Compound 3a; ${ }^{1} \mathrm{H}$ NMR (400 MHz, $\left.\mathrm{CDCl}_{3}\right)$ : $\delta 3.83(\mathrm{~s}, 3 \mathrm{H}), 6.90(\mathrm{~d}, 2 \mathrm{H}), 7.32-7.39(\mathrm{~m}, 3 \mathrm{H}), 7.52-7.56(\mathrm{~m}$, $2 \mathrm{H}), 7.54(\mathrm{~s}, 1 \mathrm{H}), 7.59(\mathrm{~d}, 2 \mathrm{H})$; Mass (EI mode): $\mathrm{m} / z$ (relative intensity) 51 (21.4), 77 (61.4), 90 (42.5), 103 (31.4), 105 (66.2), 106 (66.2), 133 (100), 213 (24.6), $319\left(\mathrm{M}^{+}, 3.2\right)$; HRMS calcd for $\mathrm{C}_{15} \mathrm{H}_{13} \mathrm{NO}_{2} \mathrm{Se}\left(\mathrm{M}^{+}\right)$319.0112, found 319.0109. Compound 3c; ${ }^{1} \mathrm{H}$ NMR $\left(400 \mathrm{MHz}, \mathrm{CDCl}_{3}\right): \delta 3.84$ (s, 3H), 6.91 (d, 2H), 7.57 (s, 1H), 7.59 (d, 2H), 7.63 (s, 4H); Mass (EI mode): $\mathrm{m} / z$ (relative intensity) 90 (32.0), 103 (22.2), 133 (100), 145 (64.9), 173 (73.0), 174 (53.9), 198 (22.0), 211 $(21.9), 213$ (44.7), $387\left(\mathrm{M}^{+}, 8.6\right)$; HRMS calcd for $\mathrm{C}_{16} \mathrm{H}_{12} \mathrm{~F}_{3} \mathrm{NO}_{2} \mathrm{Se}\left(\mathrm{M}^{+}\right)$386.9985, found 386.9981. Compound 3e; ${ }^{1} \mathrm{H}$ NMR (400 MHz, $\left.\mathrm{CDCl}_{3}\right): \delta 7.37(\mathrm{~d}, 2 \mathrm{H}), 7.44(\mathrm{~s}, 1 \mathrm{H})$, 7.45-7.55 (m, 5H), $7.58(\mathrm{~d}, 2 \mathrm{H})$; Mass (EI mode): $\mathrm{m} / \mathrm{z}$ (relative intensity) $77(21.1), 105(20.6), 137(50.0), 176$ (31.2), 215 (20.2), 217 (43.3), 246 (100), 248 (65.3), $323\left(\mathrm{M}^{+}\right.$, 4.7); HRMS calcd for $\mathrm{C}_{14} \mathrm{H}_{10} \mathrm{ClNOSe}\left(\mathrm{M}^{+}\right) 322.9616$, found 322.9626.

16. Yao, C-F.; Kao, K-H.; Liu, J-T.; Chu, C-M.; Wang, Y.; Chen, W-C.; Lin, Y-M.; Lin, W-W.; Yan, M-C.; Liu, J-Y.; Chuang, M-C.; Shiue, J-L. Tetrahedron 1998, 54, 791-822.

17. Selected spectral data of [3+2] Cycloadducts 4. Compound 4a; ${ }^{1} \mathrm{H}$ NMR $\left(400 \mathrm{MHz}, \mathrm{CDCl}_{3}\right): \delta 6.83-6.88(\mathrm{~m}, 1 \mathrm{H}), 7.08(\mathrm{~s}$, $1 \mathrm{H}), 7.14-7.40(\mathrm{~m}, 12 \mathrm{H}), 7.63-7.66(\mathrm{~m}, 2 \mathrm{H})$; Mass (EI mode): $\mathrm{m} / \mathrm{z}$ (relative intensity) 77 (69.2), 91 (82.1), 103 (28.3), 104 (100), 180 (54.2), 181 (51.2), 194 (34.4), 207 (43.2), 362 (22.2), $364\left(\mathrm{M}^{+}, 44.2\right)$; HRMS calcd for $\mathrm{C}_{20} \mathrm{H}_{16} \mathrm{~N}_{2} \mathrm{Se}\left(\mathrm{M}^{+}\right)$ 364.0479, found 364.0481. Compound $4 \mathrm{c} ;{ }^{1} \mathrm{H}$ NMR (400 $\left.\mathrm{MHz}, \mathrm{CDCl}_{3}\right): \delta$ 6.87-6.90 (m, 1H), 7.02-7.47 (m, 9H), 7.10 (s, 1H), $7.55(\mathrm{~d}, 2 \mathrm{H}), 7.63(\mathrm{~d}, 2 \mathrm{H})$; Mass (EI mode): $\mathrm{m} / \mathrm{z}$ (relative intensity) 77 (80.2), 91 (88.7), 103 (33.7), 104 (100), 172 (49.1), 173 (21.3), 194 (25.3), 248 (53.3), 249 (52.2), 275 (46.7), 430 (23.8), $432\left(\mathrm{M}^{+}, 47.8\right)$; HRMS calcd for $\mathrm{C}_{21} \mathrm{H}_{15} \mathrm{~F}_{3} \mathrm{~N}_{2} \mathrm{Se}\left(\mathrm{M}^{+}\right)$432.0353, found 432.0350. Compound 4d; ${ }^{1} \mathrm{H}$ NMR (400 MHz, $\mathrm{CDCl}_{3}$ ): $\delta$ 6.91-6.95 (m, 1H), 7.17$7.35(\mathrm{~m}, 9 \mathrm{H}), 7.21(\mathrm{~s}, 1 \mathrm{H}), 7.74(\mathrm{~d}, 2 \mathrm{H}), 8.23(\mathrm{~d}, 2 \mathrm{H})$; Mass (EI mode): $\mathrm{m} / z$ (relative intensity) 77 (66.2), 91 (67.9), 104 (100), 105 (30.5), 180 (67.8), 181 (51.5), 252 (37.1), $409\left(\mathrm{M}^{+}\right.$, 35.1); HRMS calcd for $\mathrm{C}_{20} \mathrm{H}_{15} \mathrm{~N}_{3} \mathrm{O}_{2} \mathrm{Se}\left(\mathrm{M}^{+}\right)$409.0330, found 409.0329 . 